\title{
A fuzzy inference expert system to support the decision of deploying a military naval unit to a mission
}

\author{
Giuseppe Aiello ${ }^{1}$, Antonella Certa ${ }^{1}$ and Mario Enea ${ }^{1}$ \\ ${ }^{1}$ Dipartimento di Tecnologia Meccanica, Produzione ed Ingegneria Gestionale \\ Università degli Studi di Palermo \\ 90128, Palermo, Italy \\ \{aiello, acerta and enea\}@dtpm.unipa.it
}

\begin{abstract}
Naval military units are complex systems required to operate in fixed time frames in offshore tasks where maintenance operations are drastically limited. A failure during a mission is a critical event that can drastically influence the mission success. The decision of switching a unit to a mission hence requires complex judgments involving information about the health status of machineries and the environmental conditions. The present procedure aims to support the decision about switching a unit to a mission considering that vague and uncertain information by means of fuzzy theory and emulates the decision process of a human expert by means of a rule-based inference engine. A numerical application is presented to prove the effectiveness of the approach.
\end{abstract}

Keywords: fuzzy inference, decision support system, mission reliability.

\section{Introduction}

Naval military units are complex systems required to operate in fixed time frames in offshore tasks where maintenance operations are drastically limited. The sequence of operations to be performed in a fixed time frame constitutes the mission the ship is engaged for. A failure during a mission is a critical event that in some cases can be handled on board, but that in other cases, when it affects a critical component such as an engine or steering mechanism, requires the ship to return to a port with suitable repair facilities. Critical failures may hence prevent the ship to return to the port and consequently require the ship to be towed by another vessel. In such situations, the impossibility to perform the mission tasks in the time frame assigned eventually results in a mission failure. The evaluation of the likelihood of a failures is hence an issue of primary interest for commercial and military navies, not only because it prevents the ship to perform its functions, but also because it can be a costly event and a treat for the safety of the crew. In the traditional military, in particular, units must be "operation ready," meaning they must be ready to accomplish the missions they are assigned to. The decision of switching a unit to a mission however requires complex judgments requiring information about the health status of machineries, the available resources and the environmental conditions. The health status of machineries here refers mainly to information about the availability and condition of equipment, 
resource information concerns the availability and condition of personnel; the types of training available and the training actually received, and environmental information, refers to information about the threat situations and alert conditions in which units must operate, and information about weather and ocean conditions etc.. As it frequently happens in decision problems, some of these of information can be quantified, and some are merely descriptive: purely descriptive information may however be very valuable in the decision process.

In the present paper a decision support tool is presented to assist the decision maker in the decision of deploying a military unit to a mission. A problem that frequently arises when designing a decision support tool is to represent the vagueness and uncertainty that typically affects information which cannot be handled with traditional (crisp) mathematical models. The proposed approach takes into account such vagueness and uncertainty by means of fuzzy sets and emulates the decision process of a human expert by means of a rule-based inference engine. Experts' knowledge may in fact efficiently be represented in the form of rules when fuzzy logic is employed. Rule-based expert systems, use human expert knowledge to solve real-world problems that normally would require human intelligence. Fuzzy Inference Systems (FIS) are popular computing frameworks based on the concepts of fuzzy set theory, which have been applied with success in many fields like control [1] [2], decision support [3], system identification, etc.. Their success is mainly due to their closeness to human perception and reasoning, as well as their intuitive handling and simplicity, which are important factors for acceptance and usability of the systems [4].

\section{The fuzzy rule-based expert system for decision support}

The procedure here presented, as mentioned before, aims to be a support tool to make a decision about the switching a unit to a specific mission. As stated before several parameters that influence such decision should be taken into account, this paper however aims at presenting a methodology rather than formalizing the complete decision framework, therefore three representative parameters only have been taken into account and they have been identified by interviewing some captains of military ship. Such parameters are the reliability, the distance from the closest port (in marine miles) and the conditions of the sea (ranging from 0 to 9).

The reliability of the system involved in a mission is a primary concern since, as stated before, maintenance operations are drastically limited in offshore conditions. In addition the operating conditions of systems and machineries must be considered according to the specific mission profile since only a limited number of machines are required in each mission. For this reason it is preliminarily needed to individuate the ship subsystems (propulsion, power, etc..) whose operability is required to accomplish mission tasks. Moreover, for each subsystem, the critical components must be identified and their reliability must be linked to the reliability of the entire ship according to the functional relations expressed by the reliability block diagrams (RBD). As mentioned before, the two more input parameters here proposed in addition to the reliability; are the (maximum) distance from the closest port which is a 
key parameter in estimating the likelihood of returning to port in case of failure [5] and of the sea conditions.

The FIS is applied to each subsystem by using IF-THEN rules and fuzzy operators, to determine the impact of each subsystem on the operational readiness. At the last step of the proposed procedure, by taking the minimum value among the output values, a measure of the ship operational readiness, with relation to a given mission, is supplied. The minimum operator is chosen to assure a pessimistic assessment of the likelihood to successfully perform mission tasks.

Fig. 1 shows the whole procedure to evaluate the ship operational readiness with relation to a mission.

Fig. 1-Proposed procedure block diagram

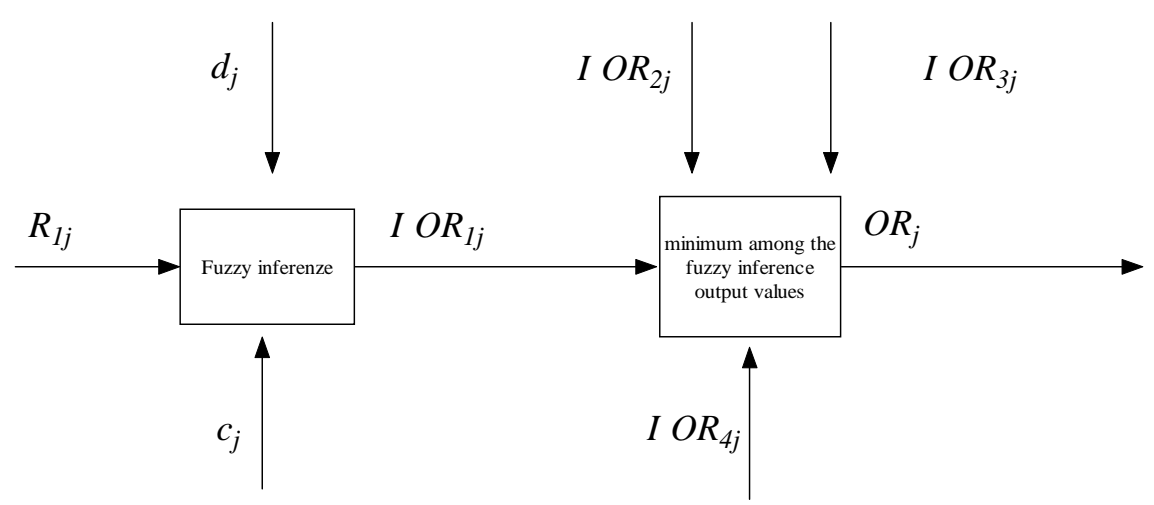

where

$i=1, \ldots, I \quad$ is the generic subsystem;

$j=1, \ldots, J$ represents the mission for which to make the decision.

$R_{i j}=$ subsystem $i$ reliability with relation to the mission $j$;

$d_{j}=$ mission distance

$c_{j}=$ conditions of the sea

$I O R_{i j}=$ impact of subsystem $i$ on the global score of the likelihood to perform mission $j$;

$O R_{j}=$ global score expressing the likelihood of performing mission

As well known, a basic fuzzy logic system is constituted four components: a rules set, a fuzzifier, an inference engine and a defuzzifier. The core of a FIS is its knowledge base, which is expressed in terms of fuzzy rules and allows for approximate reasoning [6]. The fuzzy logic system here used is a Multi Input- Single Output System (MISO), using the Mamdani implication [7] and the center of area method (COA) as defuzzifier. At first step of the inference process, it is needed to define the fuzzy set numbers to represent the crisp input value, that is the fuzzification process, which consists in assigning fuzzy linguistic variables in the universe of discourse of each input value. In particular, in this paper each input parameter is described by triangular and trapezoidal fuzzy numbers. Triangular fuzzy numbers are widely used for their simplicity and solid theoretical basis [8]. The membership 
function of a triangular fuzzy number $A$ is $\mu_{A}: \mathrm{R} \rightarrow[0,1]$ and it can be represented by the equations 1 , where $l<m<u$. Consequently, a triangular fuzzy number is fully characterized by three real numbers $(l, m, u)$. The parameter $m$ gives the maximum grade of $\mu_{A}(x)$ that is equal to $1 ; l$ and $u$ are the lower and upper bounds of the definition interval.

$$
\mu_{A}(x)= \begin{cases}\frac{x-l}{m-l} & \text { when } x \in[l, m] \\ \frac{u-x}{u-m} & \text { when } \mathrm{x} \in[m, u] \\ 0 & \text { otherwise }\end{cases}
$$

Analogously, the membership function of a trapezoidal fuzzy number $B$ is $\mu_{\mathrm{B}}$ : $R \rightarrow[0,1]$ and it can be represented by the equations $2:$

$$
\mu_{B}(x)= \begin{cases}\frac{x-l}{m-l} & \text { when } x \in[\mathrm{l}, \mathrm{m}] \\ 1 & \text { when } x \in[\mathrm{m}, \mathrm{n}] \\ \frac{u-x}{u-n} & \text { when } x \in[\mathrm{n}, \mathrm{u}] \\ 0 & \text { otherwise }\end{cases}
$$

where $l<m<n<u$.

Similarly, a trapezoidal fuzzy number is fully characterized by four real numbers $(l, m, n, u)$. The parameter $m$ and $n$ give the maximum grade of $\mu_{B}(x)$.

The next step in the fuzzy logic system is to define the possible rules arising from combining the fuzzy inputs. Rules are usually provided by a team of experts and are introduced into the FIS. Later, since the values of the assessment parameters are crisp, the fuzzifier maps the input crisp numbers into the fuzzy sets to obtain degrees of membership. The inference engine of the FIS maps the antecedent fuzzy (IF part) sets into consequent fuzzy sets (THEN part) taking into account the rules already stated. The inference process determines the fuzzy subset of the output variable for each rule by using the MIN operator (Mamdani operator) as implication operator. If more than one rule produces the same consequence, an operator must aggregate the results of these rules. In particular, the MAX operator is used. Finally, the defuzzifier maps the fuzzy output into a crisp number, which becomes the output of the fuzzy logic system, that is the impact of generic subsystem on ship operational readiness. 
As mentioned before in this case the COA method is applied and it is the most prevalent of all the defuzzification methods [9], [10].

The Fig. 2 represents the inference process.

Fig. 2. Block diagram of Fuzzy inference procedure

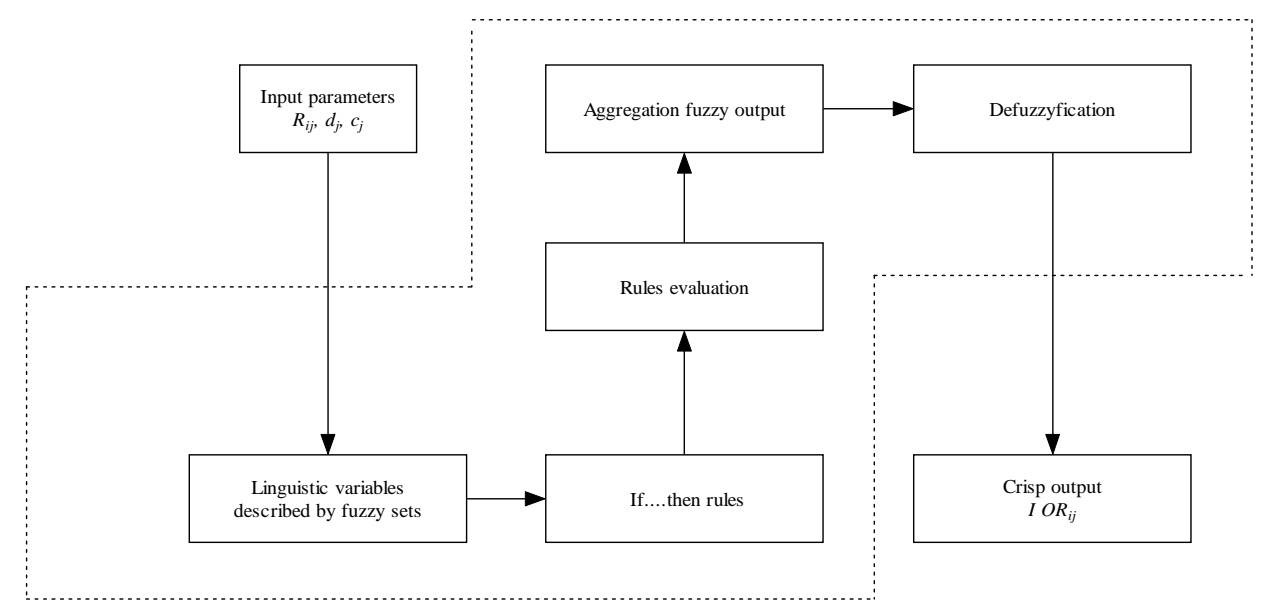

\section{Numerical application}

The proposed procedure is here applied to a simulated case with relation to a military ship. The inference process is carried out by Informs software package Fuzzy Tech. It is supposed that the ship is constituted by the following subsystem individuated as critical for the mission's success: Propulsion; Power production; Command and control; and Weapon. Such system may undergo different loading and employment conditions in different missions profiles thus resulting in different reliability values. For example, the propulsion subsystem is constituted by redundant engines and, depending on the speed required to perform the mission, they can be employed in different configurations: the reliability relation is hence different according to the specific stand-by/parallel or series configuration. The conceptual framework here considered is hence constituted by the systems reliability (according to the mission profile), the distance from the closest port and the conditions of the sea. Each input parameter has three linguistic variables (low, medium and high) described by triangular and trapezoidal fuzzy numbers, as shown in the Fig. 3, 4 and 5. Instead, the output parameter has five linguistic variables (very low, low, medium, high and very high) as shown in the Fig. 6. 
Fig. 3. Subsystem reliability

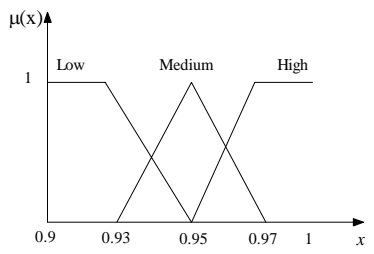

Fig. 4. Mission distance

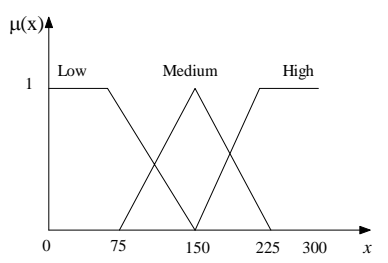

Fig. 5.Sea conditions

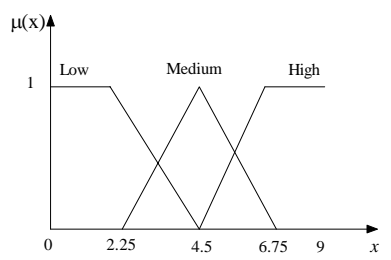

Fig. 6. Impact of each subsystem on the ship operational readiness

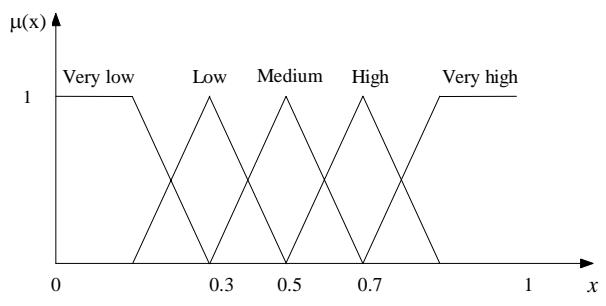

The contribution of the generic subsystem $i$ to the likelihood of performing the mission $O R_{i j}$ is expressed by values belonging to the range $[0 ; 1]$ and it can be represented, for example, by a corresponding chromatic scale. The reliability of each subsystem for the generic mission $j$, which constitutes an input to the decision system, are given in table 1 . The other input data of the mission $j$ are shown in table 2 . The set of rules individuated by the experts given in table 3.The related output value obtained by the inference process are reported in table 4 . Thus, in this simulated case, by applying the proposed procedure, that is by taking the minimum value among the output values, the ship operational readiness with relation to a given mission is 0.2029 measured in the range $[0,1]$.

\begin{tabular}{|c|c|}
\hline Subsystem & Reliability \\
\hline Propulsion & 0.98 \\
\hline Power production & 0.96 \\
\hline Command and control & 0.94 \\
\hline Weapon & 0.93 \\
\hline
\end{tabular}

Tab.1. Subsystem reliability

\begin{tabular}{|c|c|}
\hline Distance & Sea condition \\
\hline 220 & 5 \\
\hline
\end{tabular}

Tab. 2.Other input parameters 


\begin{tabular}{|c|c|c|c|}
\hline \multicolumn{3}{|c|}{ If } & \multirow{2}{*}{$\begin{array}{c}\text { then } \\
\text { Impact on ship } \\
\text { O.R. }\end{array}$} \\
\hline Reliability & Distance & Sea condition & \\
\hline Low & Low & Low & Low \\
\hline Low & Low & Medium & Very Low \\
\hline Low & Low & High & Very Low \\
\hline Low & Medium & Low & Low \\
\hline Low & Medium & Medium & Very Low \\
\hline Low & Medium & High & Very Low \\
\hline Low & High & Low & Low \\
\hline Low & High & Medium & Very Low \\
\hline Low & High & High & Very Low \\
\hline Medium & Low & Low & High \\
\hline Medium & Low & Medium & High \\
\hline Medium & Low & High & Medium \\
\hline Medium & Medium & Low & Medium \\
\hline Medium & Medium & Medium & Medium \\
\hline Medium & Medium & High & Low \\
\hline Medium & High & Low & Medium \\
\hline Medium & High & Medium & Medium \\
\hline Medium & High & High & Low \\
\hline High & Low & Low & Very High \\
\hline High & Low & Medium & High \\
\hline High & Low & High & medium \\
\hline High & Medium & Low & High \\
\hline High & Medium & Medium & Medium \\
\hline High & Medium & High & Low \\
\hline High & High & Low & High \\
\hline High & High & Medium & Medium \\
\hline High & High & High & Low \\
\hline
\end{tabular}

Tab. 3. Set of rules

\begin{tabular}{|c|c|}
\hline Subsystem & $\begin{array}{c}\text { Impact on mission } \\
\text { success. }\end{array}$ \\
\hline Propulsion & 0.4630 \\
\hline Power production & 0.455 \\
\hline Command and control & 0.2974 \\
\hline Weapon & 0.2029 \\
\hline
\end{tabular}

Tab. 4. Subsystem impact on mission likelihood. 


\section{Conclusions}

In the present paper the decision of deploying a military naval unit to a mission has been considered. Such decision generally requires a human decision process involving information about the environmental conditions, the operational status of machines etc. Such information can be hardly formalized by means of traditional (crisp) mathematical models, due to its vagueness and uncertainty, whereas such characteristics can be efficiently taken into account using approximate reasoning. In the present paper an expert decision support based upon a fuzzy inference engine is presented, which allows to take into account experts' experience in the judgments of the likelihood of a military naval unit performing a mission. The mission is described by a specific mission profile which defines the mission starting and ending time, and the set of subsystem involved The numerical application presented shows that the methodology presented may efficiently be employed to support the decision maker in the decision process providing a global score expressing the likelihood of the ship to perform the mission tasks, thus confirming the effectiveness of fuzzy inference systems in decision analysis.

\section{References}

1. Carlsson, C., Fullér, R.: Fuzzy Reasoning in Decision making and Optimization. PhysicaVerlag, New York (2002)

2. Klir, G., J., Yuan, B.: Fuzzy sets and fuzzy logic-theory and applications. Prentice Hall PTR, New Jersey (1995)

3. Bojadziev, G., Bojadziev, M.: Fuzzy logic for business, finance and management. World Scientific Publishing Co. Pte. Ltd, Singapore(1999)

4. Ross, T., J.: Fuzzy logic with engineering applications. John Wiley \& Sons, England, West Sussex (2004)

5. Christer, A., H., Lee, S., K.: Modelling Ship Operational Reliability over a Mission under Regular Inspections Author(s). The Journal of the Operational Research Society. 48 (7), 688-699 (1997)

6. Czogala, E., Leski, J.: Fuzzy and Neuro-Fuzzy Intelligent System. Physica-Verlag, New York (2000)

7. Mamdani, E., H., Assilian, S.: An Experiment in Linguistic Synthesis with a Fuzzy Logic Controller. International Journal Man-Machine Studies, 7, 1-13 (1975)

8. Pedrycz, W.: An Why Triangular Membership Functions?. Fuzzy Sets and Systems, 64 (1), 21-30 (1994)

9. Sugeno, M.: An introductory survey of fuzzy control. Inf.Sci., 36, 59-83 (1985)

10.Lee, C.: Fuzzy logic in control systems: fuzzy logic controller. Parts I and II, IEEE Trans. Syst., Man, Cybern., 20, 404-435 (1990) 